\title{
Kawasaki disease presenting as BCG scar reactivation in a five weeks old baby
}

Gamhewage NC, Kankanan Arachchige 1, Punchihewa PGM

\section{Introduction}

Kawasaki disease (KD) is an acute febrile illness of childhood of unknown origin. KD often affects children younger than five years [1]. However the incidence of $\mathrm{KD}$ in infants less than three months is very low [2].The diagnosis is made according to clinical criteria. Classic $K D$ is diagnosed based on the presence of fever lasting for five or more days, accompanied by four out of five criteria. These criteria are: bilateral bulbar conjunctival injection (without exudate), polymorphous rash, changes in oral cavity and lips, cervical lymphadenopathy and characteristic changes in extremities. Presence of two or three of above criteria associated with fever lasting five or more days constitutes incomplete KD [1]. Although BCG scar reactivation is not included as diagnostic criteria, it is a useful clinical finding which aids in the diagnosis of $K D$ in infants [3]. Cytokines like interleukin-16 and tumor necrosis factor- $\alpha$ has been detected in elevated levels at the BCG inoculation site [4].

\section{Case report}

5 week old baby boy presented with high grade fever, poor feeding and irritability for two days duration. He is the second baby of healthy nonconsanguinous parents and had an uneventful antenatal and perinatal period. He was exclusively breast fed and was thriving well. Examination revealed an ill baby with congested bulbar conjunctivae without exudate, erythematous oral mucosa and lips, bilateral pedal oedema and BCG scar reactivation (figure 1). No lymphadenopathy or skin rashes were noted. There was no organomegaly. Cardiovascular system and respiratory system examination was clinically normal. No focal neurological signs were present.

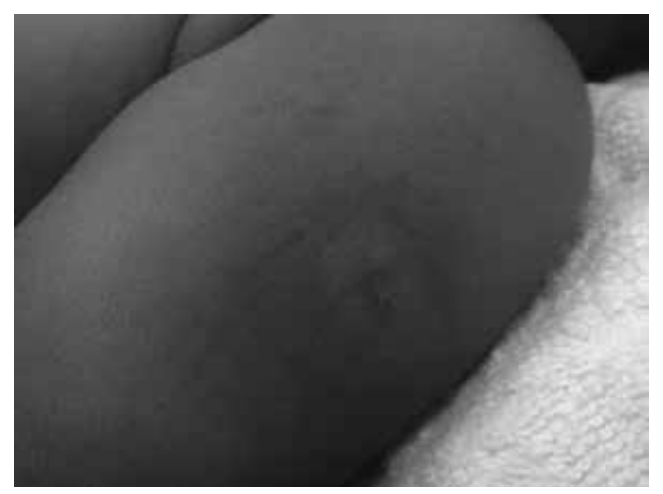

Figure 1: BCG reactivation scar

Lady Ridgeway Children Hospital
Investigations revealed neutrophil leukocytosis with normal platelet count and haemoglobin level. There were markedly elevated inflammatory markers, hypoalbuminemia, and sterile pyuria. 2 D echocardiogram was normal. Blood culture and urine culture was sterile. As there were three clinical criteria, elevated inflammatory markers and three supplemental criteria (hypoalbuminemia, sterile pyuria, white blood cells more than $15,000 / \mathrm{mm} 3$ ) diagnosis of incomplete KD was made.

Intravenous Immunoglobulin was administered on day 6 of illness together with high dose aspirin for which baby showed a marked response within forty-eight hours.

He recovered from the acute stage successfully without developing coronary artery involvement. He was found to be having typical peeling off of extremities on follow up.

\section{Discussion}

Kawasaki disease is the commonest acquired heart disease in childhood [1]. The long term squeal includes coronary aneurysms which can lead to myocardial infarction in young adults. Cardiovascular complications are most common in infants [2].

A single dose of intravenous immunoglobulin should be administered within ten days or later in a patient meeting the clinical criteria of KD. Appropriate treatment reduces the risk of developing coronary aneurysms from approximately $25 \%$ to less than $5 \%$.

Diagnosing Kawasaki disease is a challenge in young infants as there is lack of specific diagnostic tests. Reactivation of BCG scar is an early sign suggestive of $\mathrm{KD}$. This is especially important among children younger than 5 years. It is reported to be observed in more than $50 \%$ of infants with KD [3].Further, studies has shown that BCGitis is seen more prevalently than cervical lymphadenopathy and rash [4]. Therefore, presence of BCG reactivation is a specific physical sign which aids in making an early diagnosis of KD.

Paediatricians should be vigilant about the possibility of young infants getting Kawasaki disease in order to commence specific treatment early to prevent serious cardiovascular complications. We would like to highlight the importance of BCG reactivation as an important 
physical sign in KD in infants.

\section{References:}

1. Saguil A, Fargo M, Grogan S. Diagnosis and management of kawasaki disease. American family physician. 2015 Mar 15;91(6). http://www.aafp.org/ afp/2015/0315/p365.html

2. Yeom JS, Woo HO, Park JS, Park ES, Seo JH, Youn HS. Kawasaki disease in infants. Korean J Pediatr.2013 Sep; 56(9): 377-382.

3. Seo JH, Yu JJ, Ko HK, Choi HS, Kim YH, Ko JK. Diagnosis of incomplete kawasaki disease in infants based on an inflammation at the bacille calmette-guérin inoculation site. Korean Circ J. 2012 Dec;42(12):8239. doi: 10.4070/kcj.2012.42.12.823. Epub 2012 Dec 31.

4. Rezai MS, Shahmohammadi S. Erythema at BCG inoculation site in Kawasaki disease patients. Mater. Soc. 2014;26(4):256-60. doi: 10.5455/ msm.2014.26.256-260. 